\title{
Intensity dependent geminate recombination in water
}

\author{
Frederick H. Long ${ }^{1}$, Hong Lu, Xuelong Shi and Kenneth B. Eisenthal ${ }^{2}$ \\ Department of Chemistry, Columbia University. New York, NY 10027, USA
}

Received 10 July 1991

\begin{abstract}
We have observed an intensity dependent geminate recombination of electrons in neat water. Since the recombination dynamics occurs on a time scale comparable to the solvation dynamics, the kinetics characteristic of an isosbestic wavelength are obscured. We have hypothesized that this intensity-dependent geminate recombination is due to the existence of two mechanisms for electron production with different characteristic thermalization distances. These results clarify the discrepancy between experiments at higher intensities and those at lower intensities.
\end{abstract}

\section{Introduction}

The equilibrium and dynamic properties of electrons in liquids is a subject of longstanding and continuing interest in chemical physics [1-43]. Early studies established the absorption spectra of the equilibrium solvated electron in water and other polar liquids [5]. With the development of time-resolved pulse radiolysis the dynamics of electron solvation in alcohols could be investigated, and yielded some unexpected results. It was found that an IR absorbing precursor, callcd the presolvated or wet clectron, i.e. a localized but not fully solvated electron appeared prior to the development of the fully equilibrated solvated electron spectrum [10,12]. The surprising finding was that the spectrum of the initially observed wet electron did not spectrally shift in a continuous way with time from its initial IR spectrum to the final visible spectrum of the fully solvated electron, as a dielectric continuum model predicts $[10,12,17]$. This led to the development of what has come to be called the two-state model, i.e. that there are principally two electron species present in the early stages of electron solvation, the initial IR absorbing wet electron which decays directly into the other electron species, the visible absorbing solvated electron $[10,12]$.

1 IBM Graduate Student Fellow.

2 To whom correspondence should be addressed.
Although laser picosecond time-resolved studies were possible for alcohols $[13,15]$, and supported the radiolysis results, it was not until the development of subpicosecond lasers that the ultrafast solvation dynamics in water could be studied $[14,19,40]$. A key issue in the water solvation dynamics is whether the two-state model is applicable. To this end a study was initiated to determine if there is an isosbestic wavelength, i.e. the wavelength at which the absorption coefficients of the wet and solvated electron are equal [40]. The characteristic kinetics at the isosbestic wavelength is a dircet test of the two-species model [40]. With the discovery of an isosbestic wavelength at $820 \mathrm{~nm}$ strong support of the two-state model, in agreement with the initial conjecture by Migus et al. [19], was obtained [40]. However there is continuing interest and some controversy concerning the existence of the isosbestic wavelength. Due to its importance in establishing the two-state model we have carried out further experiments which clarify the discrepancy between our results and those of Gaudel and co-workers [43]. We have discovered that there is an intensity dependence to the geminate electron recombination kinetics, which in a given intensity range introduces a very fast decay that obscures the observation of the rise time kinetics at the isosbestic wavelength. This intensity-dependent decay that we have found explains the apparent discrepancies alluded to above. Although the identification of the origin of the in- 
tensity-dependent recombination is not necessary to reconcile these differences it is of interest to examine and at least speculate about this finding to better understand electron dynamics in aqueous solutions. A plausible starting point would assume that the thermalization distances are intensity dependent and thus the recombination dynamics observed is intensity dependent. One possibility that we will explore is the presence of two mechanisms of electron generation, which have different intensity dependences. In this paper we describe experiments relevant to these issues.

\section{Experimental}

The experiments performed were transient absorption measurements using an amplified collidingpulse-mode-locked (CPM) laser. Our UV pump pulses were generated with a $200 \mu \mathrm{m}$ BBO (beta-bariumborate) crystal. Temporally broader pulses are generated by a $1 \mathrm{~mm}$ KDP (potassiumdihydrogenphosphate) crystal, which we previously used $[38,39]$. The fundamental pulse width was found to be $80 \mathrm{fs}$ fwhm, using a Gaussian pulseshape. To characterize the pump-probe pulse widths, i.e. the system response function, a cross-correlation technique was used. By cross-correlations of the UV pulse and the fundamental, the UV pulse was determined to be $150 \mathrm{fs}$ fwhm. By difference frequency generation, the cross-correlation of the $820 \mathrm{~nm}$ probe and the UV pump was found to be a symmetric Gaussian with the $820 \mathrm{~nm}$ pulse $170 \mathrm{fs}$ fwhm in duration. A $100 \mu \mathrm{m} \mathrm{KDP}$ crystal was used in these measurements in order to accurately measure the pulsewidths. In order to minimize group velocity dispersion between the pump and probe a $200 \mu \mathrm{m}$ cuvette was used in the experiments below. From these measurements we have found that the signal from $n$-octane is not instrument-limited as we and others had found using broader pulses, but rather has a formation time of approximately $150 \mathrm{fs}[19,40]$.

\section{Results and discussion}

From a comparison of the probe signals at $820 \mathrm{~nm}$ at different pump intensities, figs. $1 \mathrm{a}, \mathrm{lb}$ and $\mathrm{lc}$, we
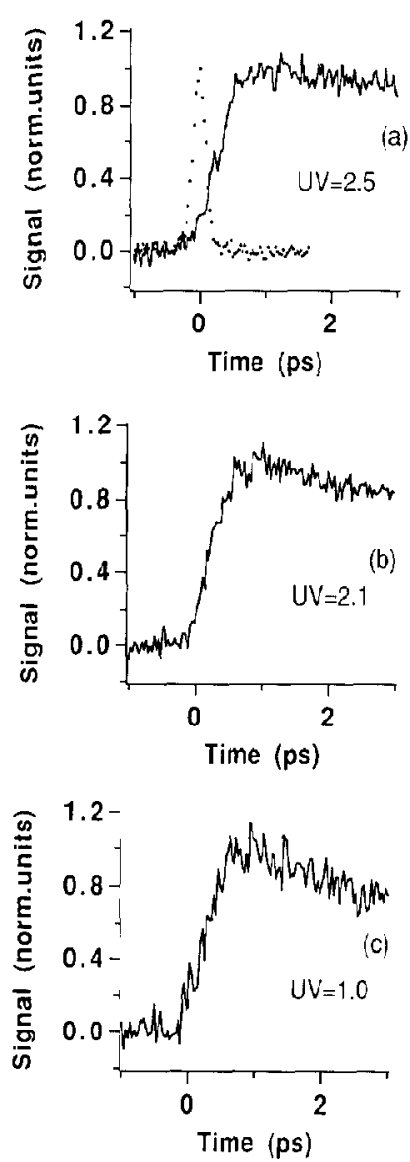

Fig. 1. The dynamics of the electron probed at $820 \mathrm{~nm}$ as a function of UV pump intensity. An increased decay rate is found at low $U V$ powers $\left(U V=2.5,1 / k_{\mathrm{R}} \geqslant 15 \mathrm{ps} ; \mathrm{UV}=2.0,1 / k_{\mathrm{R}}=8 \pm 1.5\right.$ $\left.\mathrm{ps} ; U V=1.0,1 / k_{\mathrm{R}}=4.5 \pm 1.5 \mathrm{ps}\right)$. In contrast the rise time of the wet clectron was found to be intensity independent. The dots in (a) are the cross-correlation of the pump and the probe.

see that there is a well-defined intensity effect on the observed decay within the first few picoseconds. For the higher intensity pump we note that the probe signal shows no decay initially, fig. 1a, whereas a significant decay is observed when the intensity of the pump is lowered by a factor of 2.5 , fig. $1 c$. Thus at higher intensities the kinetic characteristic of an isosbestic wavelength are found at $820 \mathrm{~nm}$, i.e. a rise due to the formation of the wet electron with no change in the kinetics as the transition to the solvated electron occurs. This absence of change in the absorption kinetics at the isosbestic wavelength is due to the fact that the absorption coefficients of the two species are equal at the isosbestic wavelength. If there 
were many different wet electron species with lifetimes on the time scale of the transition to the solvated electron then there would be no isosbestic wavelength nor the characteristic isosbestic kinetics, since they could not all have the same absorption coefficients at any given wavelength. It should be noted as well that the "flat" part of the isosbestic kinetics is based on the absence of a recombination or other loss process on the time scale of the solvation. The real kinetics cannot of course be perfectly flat since the electron undergoes geminate recombination at longer times [38-41]. Geminate recombination of electrons has also been observed in non-polar liquids [44-47]. Contrasted with this behavior we note at lower intensities, fig. Ic, that there is a marked decay following the initial rise. The decay seen at the lower intensity obscures the "flat" region expected for the kinetics at an isosbestic wavelength. We attribute this decay to geminate recombination of the solvated electron. In other words the solvated electron is undergoing a recombination on a time scale that is not sufficiently slower than its formation time to permit a readily observed separation of these processes in time.

To analyze the experimental results obtained at the lower intensities where geminate recombination is important in the first few picoseconds, we have included this electron loss process in the kinetic model. Since the decay that affects the leveling off at the isosbestic wavelength $(820 \mathrm{~nm})$ occurs out to $4 \mathrm{ps}$ and beyond we can reasonably treat the recombination of the solvated electron and neglect the wet electron recombination in this long time domain. The wet electron has effectively disappeared in $2-3$ ps as established by the unchanging solvated electron spectrum after this time.

A two-state model that includes electron loss due to recombination with the other products of the electron photogeneration, namely $\mathrm{H}_{3} \mathrm{O}^{+}$and $\mathrm{OH}$, can be written as follows,

$\mathrm{e}_{\mathrm{qf}}^{-} \stackrel{h_{1}}{\longrightarrow} \mathrm{e}_{\mathrm{wet}}^{-}$,

$\mathrm{e}_{\text {wet }}^{-} \stackrel{k_{2}}{\longrightarrow} \mathrm{e}_{\text {solv }}^{-}$,

$\mathrm{e}_{\text {wet }}^{-}+\left\{\begin{array}{c}\mathrm{H}_{3} \mathrm{O}^{+} \\ \mathrm{OH}\end{array}\right\} \stackrel{k_{2}^{\prime}}{\longrightarrow}$ loss,

$\mathrm{e}_{\text {solv }}^{-}+\left\{\begin{array}{c}\mathrm{H}_{3} \mathrm{O}^{+} \\ \mathrm{OH}^{-}\end{array}\right\} \stackrel{k_{\mathrm{R}}}{\longrightarrow}$ loss where $e_{\mathrm{qf}}^{-}$is the quasifree clectron. We note that the kinetics of geminate recombination is in general not exponential; however, at short times there is a sufficiently small amount of recombination that it can be approximated as an exponential.

At high intensities we found the solvated electron recombination rate constant $k_{\mathrm{R}}$ is slower than (15 ps $)^{-1}$. This is indeed slow compared with the $2-3$ ps for the completion of the electron solvation and thus yields the characteristic isosbestic "flat" leveling off kinetics, fig. 1. At low intensities, however, $k_{\mathrm{R}}$ is at least three times larger having the value of $(4.5 \pm 1.5$ ps $)^{-1}$. Therefore the recombination is sufficiently fast to obscure the expected isosbestic behavior. We have found that broader, less intense pulses are typically produced with a $1 \mathrm{~mm} \mathrm{KDP}$ crystal, as was used by Gaudel et al. $[19,41,43]$. We believe this is the source of the discrepancy between our results (high intensity) and that of Gaudel et al. (low intensity).

A result of some interest is our observation that the appearance time of the solvated electron, $\left(k_{2}+k_{2}^{\prime}\right)^{-1}$ measured at $625 \mathrm{~nm}$ has the same value of $540 \pm 30 \mathrm{fs}$ at low and higher intensities. This suggests that the recombination loss of the wet electron, $k_{2}^{\prime}$, which should be intensity dependent, is not important on the time scale of the wet electron lifetime. A further result of interest is the observation that the rise time kinetics, $k_{1}$, at the isosbestic wavelength $(820 \mathrm{~nm})$ was the same $(300 \pm 30 \mathrm{fs})$ at both the low and high intensities. These results on the kinetics of the wet and solvated electron show that the formation of the wet electron, $k_{1}$, and the transition of the wet electron to the solvated electron, $k_{2}$, are not dependent on intensity. From this we conclude that the electron solvation dynamics portion of the kinetics is the same, regardless of the mechanisms responsible for the intensity-dependent generation and recombination dynamics. It should also be noted that the rate constants obtained by fitting the kinetic data to the two-state model with recombination was the same at 820 and $625 \mathrm{~nm}$, i.e. independent of the probe wavelength. This was found to be the case both in the low and higher intensity experiments.

\section{Possible model for intensity-dependent kinetics}

Let us now consider a possible model to explain 
the observed intensity-dependent kinetics. This model is based on two different mechanisms for electron generation, which produce different average thermalization distances from the parent ions. Due to their different intensity dependences they are responsible for the observed intensity-dependent geminate recombination kinetics. The first mechanism is the multiphoton excitation of water above its photoionization threshold at $8.5 \mathrm{eV}[8,35]$. The ultraviolet photons comprising the femtosecond excitation pulse are at $4 \mathrm{eV}$, thus three photons are required to exceed the photoionization threshold of liquid water. The electron will therefore have at most $3.5 \mathrm{eV}$ of excess energy, which is likely to result in a thermalization distance that greatly exceeds the Onsager distance of about $7 \AA[38,39,41,48]$. The Onsager distance is the distance at which the Coulomb electron-cation interaction energy is equal to the thermal energy $k T$. At thermalization distances greater than this there is a higher probability of electron escape and conversely at shorter distances it is more likely for the electron to recombine with the parent cation.

At the lower intensity, fig. $1 \mathrm{c}$, we note that the electron absorption decay about $20 \%$ in the first few picoseconds, in contrast to the absence of a clear decay at the higher intensity, fig. la. We attribute this more rapid geminate recombination to a two-photon electron generation process. It was experimentally established by two different groups more than twenty years ago that electrons are produced upon the production of electronically excited states in liquid water $[6,7]$. The measured threshold for this process was found to be about $6.5 \mathrm{eV}$, which would require two photons in the experiments reported here. The details of the mechanism have not been established at this time; however, a model has been proposed that involves a water molecule in an excited state reacting with a water molecule in its ground electronic state $[6,7,35]$. The reaction, thought to occur by a proton transfer between the water molecules, produces a solvated electron, a solvated hydronium ion and a hydroxyl radical:

$$
\mathrm{H}_{2} \mathrm{O}^{*}+\mathrm{H}_{2} \mathrm{O} \rightarrow \mathrm{e}^{-}+\mathrm{H}_{3} \mathrm{O}^{+}+\mathrm{OH}^{\cdot} \text {. }
$$

Based on a thermodynamic cycle the energy required for two water molecules in the liquid state to undergo the reaction shown above is about $5.7 \mathrm{eV}[7,35]$.
However, it must be noted that the energies of the various parts of the cycle are not all experimentally known and require theoretical estimates. Nevertheless this is the only known model that can rationalize the generation of electrons with a weak light source having photons at a threshold energy of about 6.5 $\mathrm{eV}$. In this reaction, which produces an $\mathrm{e}^{-}, \mathrm{H}_{3} \mathrm{O}^{+}$, and $\mathrm{OH}^{\circ}$, there is significant nuclear motion involved and it would be expected that the excess $1.5-$ $2 \mathrm{eV}$ above the two-photon energy of $8 \mathrm{eV}$, does not end up exclusively or even chiefly in the electron's kinetic energy. The effect of this indirect process of electron generation is that the thermalized electron can be much nearer to the cation and hydroxyl radical, with which it can recombine, than occurs in the three-photon ionization mechanism.

In our experiments it is likely that the indirect twophoton mechanism and the thrce-photon mechanism are both occurring. Their relative importance is determined by the UV laser intensity. At higher intensities the three-photon and two-photon mechanisms can occur whereas at lower intensities the twophoton mechanism dominates the electron generation ${ }^{\#}$. At the higher intensities the three-photon cross-section $\sigma_{3}$ times the laser intensity $4 \times 10^{12} \mathrm{~W}$ / $\mathrm{cm}^{2}$ is likely to be larger than the two-photon crosssection $\left(9 \times 10^{-52} \mathrm{~cm}^{4} \mathrm{~s}\right)[16]$. Note that the threephoton ionization can be a direct three-photon process as well as a two-plus-one-photon process. In fact from gas phase studies it is known that $(2+1)$-multiphoton ionization is a more efficient means of producing electrons than direct 3-proton ionization [50]. By increasing the intensity in steps over a range of 2.5 we observe a significant decrease in the geminate recombination rate. The decay kinetics for intermediate intensities are shown in fig. $1 \mathrm{~b}$. Although we do not know if this model is the one that is responsible for the intensity-dependent kinetics, it does appear to be consistent with the results of our experiments and is consistent with the finding that a $6.5 \mathrm{eV}$ photon produces electrons in neat water. An alternate explanation of the observed intensity-dependent geminate recombination is that at high pump

\#- We have not included the possibility that the observed intensity dependence arises from a four-photon ionization. The cross-section would typically be too small by several orders of magnitude to contribute to the electron generation mechanism at our intensities [49]. 
intensitics the ionization potential is changed significantly by a Stark-effect-like mechanism. Such an effect, above threshold ionization, has been studied in gas phase experiments using picosecond and subpicosecond light pulses [51]. We estimate that under the conditions of our experiments the pump pulse intensity is approximately an order of magnitude too low, to significantly shift the vertical ionization potential of neutral water.

Assuming for the moment that the two- and threephoton model is applicable we can now try to understand the implications of finding the same values for the wet electron formation time constant $k_{1}$ and the same values for the wet to solvated constant $k_{2}$ at the different intensities. It is not surprising that $k_{2}$ is the same for the two mechanisms since we interpret $k_{2}$ to be an excited state relaxation process from the lowest excited electron state (wet electron) to its ground state (solvated electron) [27,33,34,37,40, 421. Unless the proximity of the wet electron to the $\mathrm{H}_{3} \mathrm{O}^{+}$and $\mathrm{OH}$ species perturbs this relaxation, we should expect the excited state relaxation rate constant $k_{2}$ to be the same for the two- and three-photon mechanisms. However, finding the same value for $k_{1}$ for the two mechanisms is a hit more interesting. In the two-photon process we do not expect to produce energetic quasi-free electrons as is the case in the three-photon ionization process. How then can the formation time of the wet electron be the same for the two mechanisms? One possibility is that there is a common rate limiting step for the two mechanisms. Let us interpret $k_{1}$ as being determined chiefly not by the localization of the quasi-free electron but rather by the internal conversion kinetics from high excited electron states, perhaps a d-like state, to the wet electron ( $p$-like) state. Furthermore we would expect that the relaxation from upper states to the lowest excited state, i.e. the wet electron state, would be expected to be the same regardless of how the upper excited states were initially produced. In other words $k_{1}$ is not determined by the localization of the quasi-free electron but rather by the rclaxation from upper excited states of the localized electron to the first excited state, i.e. the wet electron. As with the three-photon mechanism the two-photon mechanism has sufficient excess energy to produce an upper excited state of the electron. The decay of this initially produced upper state to first excited state would therefore occur at a similar rate to the threephoton process.

\section{Conclusion}

We have carried out studies of electron solvation and geminate recombination in neat water using improved pulse characterization, which clarify the controversy concerning the existence of an isosbestic wavelength in the solvation dynamics. The origin of the disparate results is an intensity-dependent geminate recombination that obscures the existence of an isosbestic wavelength at lower pump intensities. We have hypothesized that this intensity-dependent geminate recombination is due to the existence of two mechanisms for electron production with different characteristic thermalization distances. This hypothesis is consistent with the known radiation chemistry of liquid water.

\section{Acknowledgement}

We would like to thank the Air Force Office of Scientific Research and the National Science Foundation for their generous financial support.

\section{References}

[1] W. Weyl, Ann. Phys. 121 (1864) 601.

[2] J. Franck and G. Scheibe, Z. Physik. Chem. A 139 (1928) 22.

[3] L.D. Landau, Phys. Z. Sowjetunion 3 (1933) 664.

[4] J. Jortner, Mol. Phys. 5 (1962) 257.

[5] E.J. Hart and J.W. Boag, J. Am. Chem. Soc. 84 (1962) 4090.

[6] U. Sokolov and G. Stein, J. Chem. Phys. 44 (1966) 3329.

[7] J.W. Boyle, J.A. Ghormley, C.J. Hochanadel and J.F. Riley, J. Phys. Chem. 73 (1969) 2886.

[8] P. Delahay and K. von Berg, Chem. Phys. Letters 83 ( 1981 ) 250.

[9] K.H. Schmidt and W.I. Buck, Science 15 (1966) 70; H.A. Schwartz, J. Phys. Chem. 73 (1969) 1928.

[10] M.J. Bronskill, R.K. Wolff and J.W. Hunt, J. Chem. Phys. 53 (1970) 420 l.

[11] P.M. Rentzepis, R.P. Jones and J. Jortner, J. Phys. Chem. 59 (1973) 766.

[12] W.J. Chase and J.W. Hunt, J. Phys. Chem. 79 (1975) 2835. 
[13] Y. Wang. M.K. Crawford, M.J. McAuliffe and K.B. Eisenthal, Chem. Phys. Letters 74 (1980) 160.

[14] J.M. Wiesenfeld and E.P. Ippen, Chem. Phys. Letters 73 (1980) 47.

[15] G.A. Kenney-Wallace and C.D. Jonah, J. Phys. Chem. 86 (1982) 2572.

[16] D.N. Nikogosyan, A.A. Oraevsky and V.I. Rupasov, Chem. Phys. 77 (1983) 131.

[17] H. Frohlich, Theory of dielectrics (Oxford Univ. Press, Oxford, 1986).

[18] Farhataziz and M.A.J. Rodgers, eds., Radiation chemistry principles and applications ( $\mathrm{VCH}$, Weinheim 1987); G.R. Freeman, ed., Kinetics of inhomogeneous processes (Wiley, New York, 1987).

[19] A. Migus, Y. Gaudel, J.L. Martin and A. Antonetti, Phys. Rev. Letters 58 (1987) 1559.

[20] W.M. Bartzak, M. Hiltzer and J. Kroh, J. Phys. Chem. 91 (1987) 3834

[21] A.C. Chernovitzand C.D. Jonah, J. Phys. Chem. 92 (1988) 5946.

[22] J. Schnitker, K. Motakabbir, P.J. Rossky and R. Friesner, Phys. Rev. Letters 60 (1988) 456.

[23] A. Wallqvist, G. Martyna and B.J. Berne, J. Phys. Chem. 92 (1988) 1721 .

[24] M. Maroncelli and G.R. Fleming, J. Chem. Phys. 89 (1988) 5044.

[25] R.N. Barnett, U. Landman and A. Nitzan, J. Chem. Phys. $91(1989) 5567$.

[26] P.J. Rossky and J. Schnitker, J. Phys. Chem. 93 (1989) 6965.

[27] P.J. Rossky and J. Schnitker, J. Phys. Chem, 92 (1988) 4277.

[28 ] A.K. Pikaev, The solvated electron in radiation chemistry (Keter, New York, 1971).

[29] M. Sprik and M.L. Klein, J. Chem. Phys. 91 (1989) 5665.

[30] T. Goulet and J.P. Jay-Gerin, J. Phys. Chem. 92 (1989) 6871.

[31 ] R.N. Barnett, U. Landman and A. Nitzan, J. Chem. Phys. 90 (1989) 4413.

[32] C.V. Coe, G.H. Lee, J.G. Eaton, S.T. Arnold, H.W. Sarkes, K.H. Bowen, C. Ludewigt and D.R. Worsnop, J. Chem. Phys. 92 (1990) 3890;
P.J. Campagnola, L.A. Posey and M.A. Johnson, J. Chem. Phys. 92 (1990) 3243.

[33] R.N. Barnett, U. Landman and A. Nitzan, J. Chem. Phys. 93 (1990) 8187;

R.N. Bamett, U. Landman, G. Makov and A. Nitzan, J. Chem. Phys. 93 (1990) 6226.

[34] M.C. Messmer and J.D. Simon, J. Phys. Chem. 94 (1990) 1220.

[35] P. Han and D.M. Bartels, J. Phys. Chem. 94 (1990) 5824.

[36] I. Rips and R. Silbey, J. Chem. Phys. 94 (1991) 4495.

[37] B. Space and D.F. Coker, J. Chem. Phys. 94 (1991) 1976.

[38] H. Lu, F.H. Long, R.M. Bowman and K. B. Eisenthal, J. Phys Chem. 93 (1989) 27.

[39] F.H. Long, H. Lu and K.B. Eisenthal, Chem. Phys. Letters $160(1989) 464$.

[40] F.H. Long, H. Lu and K.B. Eisenthal, Phys. Rev. Letters 64 (1990) 1490;

H. Lu, F.H. Long and K.B. Eisenthal, J. Opt. Soc. Am. 7 (1990) 1511.

[41] Y. Gaudel, S. Pommeret, A. Migus, N. Yamada and A. Antonetti, J. Am. Chem. Soc. 112 (1990) 2925.

[42] F. Webster, J. Schnitker, M. Freidrichs, R. Freisner and P.J. Rossky, Phys. Rey. Letters 66 (1991) 3172.

[43] Y. Gaudel, S. Pommeret, N. Yamada, A. Migus and A. Antonetti, Ultrafast phenomena, Vol. 7, Femtosecond dynamics of a single electron transfer in aqueous media (Springer, Berlin, 1990).

[44] C.L. Braun and T.W. Scott, J. Phys. Chem. 91 (1987) 4436.

[45] A.M. Brearly and D.B. McDonald, Chem. Phys. Letters 155 (1989) 83.

[46] R.M. Bowman, H. Lu and K.B. Eisenthal, J. Chem. Phys. 89 (1988) 606.

[47] Y. Hirata and N. Mataga, J. Phys. Chem. 95 (1991) 1640.

[48] L. Onsager, Phys. Rev. 54 (1938) 554.

[49] J.H. Eberly, in: Advances in chemical physics, Vol. 73, eds. J.O. I Iirshfelder, R.E. Wyatt and R.D. Coalson (Wiley, New York, 1989) p. 803.

[50] G. Meijer, J.J. ter Meulen, P. Andressen and A. Bath, J. Chem. Phys. 85 (1986) 6914.

[51] R.R. Freeman, P.H. Bucksbaum and T.J. McIlrath, IEEE J. Quantum Electron. QE-24 (1988) 1461. 